\title{
Energy-efficient MAC in Wireless Body Area Networks
}

\author{
Jingjing Yuan ${ }^{1}$, Changle $\mathrm{Li}^{1,2}$, and $\mathrm{Wu} \mathrm{Zhu}^{1}$ \\ ${ }^{1}$ State Key Laboratory of Integrated Service Networks, Xidian University \\ Xi'an, Shaanxi, 710071 China \\ ${ }^{2}$ National Mobile Communications Research Laboratory, Southeast University \\ Nanjing, Jiangsu 210096, China \\ e-mail: yuanjing0923@163.com,clli@mail.xidian.edu.cn,wu_zhu@126.com
}

\begin{abstract}
Recently wireless body area network (WBAN) draws more and more attentions because of its benefits especially in health monitoring. As the sensor nodes in WBAN are batterypowered, energy efficiency is the top concern in the medium access control (MAC) protocol design. Therefore, in this paper, we propose an Enhanced MAC (EMAC) protocol which integrates relay with dynamic power control mechanism to save energy consumption. On one hand, the protocol selects a relaying node for the node which may be energy shortage to prolong its lifetime, and then the network topology is switched from one-hop to multi-hop. Accordingly, the superframe structure is modified. On the other hand, for further energy saving, dynamic power control algorithm is performed whenever sensor nodes have data packets to transmit. Performance evaluations are conducted and the simulation results show that the EMAC significantly prolongs the network lifetime compared with IEEE 802.15.4 MAC.
\end{abstract}

Keywords-WBAN; MAC; relay; power control; energy efficiency

\section{INTRODUCTION}

Wireless body area networks (WBANs) consist of a series of low-power, intelligent sensor nodes distributed on, in or around the human body for physical condition and the surrounding environment monitoring. As a special wireless sensor network which is human-centered, WBAN has many distinctive features distinguishing from other wireless sensor network [1] and many challenges are faced in the medium access control (MAC) protocol design [2]. One of the greatest challenges is energy efficiency. Especially for the nodes which are implanted into the human body, energy is more precious because that battery replacement or recharging is impractical. Therefore, to minimize the energy consumption of the sensor nodes is the prime consideration in the MAC protocol design.

Existing researches on the MAC layer are mostly focused on maximizing the sensor node's lifetime. In [3], a crosslayer based battery-aware TDMA MAC protocol was proposed for healthcare applications in WBANs. Considering the channel characteristics and the queuing management, the protocol prolonged the battery lifespan of the sensor nodes. In [4], a protocol based on TDMA and/or contention mechanisms was also proposed to enhance energy efficiency. However, these protocols were one-fold network formation - one-hop topology which may lead to unreliability and energy waste. As a result, some research began to consider constructing a reliable link between nodes through multi-hop connection. In [5], a minimum spanning tree (MST) is constructed by the coordinator for the implanted BAN based on each node's battery status as well as the distance. By choosing proper relaying nodes, the network lifetime was effectively prolonged. One protocol of choosing relaying nodes to save energy consumption was also presented in [6]. Therefore, to prolong the network lifetime effectively, the proposed protocol in this paper also employs relaying nodes.

On the other hand, an increasing study focuses on the distinct benefit of the power control mechanism on power saving in WBANs. In [7], an energy management mechanism named dynamic postural position inference (DPPI) is explored for WBANs. DPPI performed adaptive body posture inference for optimal power assignments to ensure a balance between energy consumption and packet loss on links. Another dynamic power control strategy to save energy consumption was proposed in [8]. Consequently, considering that power control mechanism can not only improve the reliability of the link but also has the ability to save energy consumption which conforms to the demands in WBANs, it is a good way to perform dynamic power control for data packet transmission.

As a result, in this paper, we propose an Enhanced MAC (EMAC) protocol based on IEEE 802.15.4 multi-hop superframe structure [9] to prolong the network lifetime from the aspects of relay and dynamic power control. As far as we know, it is the first time to design the MAC protocol integrating both of the relay and dynamic power control. The rest of the paper is organized as follows. In Section 2, the paper gives an overview of IEEE 802.15.4 MAC. Then, we will discuss the details of the EMAC protocol. Performance evaluation of the EMAC is described in Section 4. Finally, the conclusions of the paper are drawn in the last section.

\section{IEEE 802.15.4 MAC OVERVIEW}

IEEE 802.15.4 standard defines the specifications for physical layer (PHY) and MAC layer of a low-rate wireless personal area network (WPAN). It may operate in the star topology and the peer-to-peer topology, as illustrated in Fig. 1 [9]. In either of the topologies, beacon-enabled mode and nonbeacon-enabled mode could be adopted in the network. In the beacon-enabled mode, there are two kinds of 
superframe structure defined in IEEE 802.15.4. One is for one-hop star network as shown in Fig. 2, the other one is for multi-hop network as shown in Fig. 3.

For one-hop superframe structure, the time is divided into equivalent periodic interval, each of which consists of an active portion and an inactive portion. The active portion is partitioned into 16 slots evenly and consists of three parts: a beacon, a contention access period (CAP) and a contention free period (CFP). In CAP, nodes employ CSMA/CA mechanism to access the channel. To support time critical data application, the PAN coordinator can reserve one or more guarantee time slots (GTSs) for nodes without contention with other devices and these GTSs consist of the CFP period. The beacon interval (BI) is determined by the parameter of beacon order (BO) and the superframe duration (SD) by superframe order (SO), as shown in Fig. 2.

For the multi-hop superframe structure, both of the PAN coordinator and any ordinary coordinator may periodically transmit its own beacons. A coordinator that is not the PAN coordinator shall maintain the timing of both the superframes in which its coordinator transmits a beacon (the incoming superframe) and the superframes in which it transmits its own beacon (the outgoing superframe). The relationship between incoming and outgoing superframes is illustrated in Fig. 3 [9].

\section{MAC PROTOCOL DESIGN}

\section{A. EMAC Overview}

The EMAC employs not only relaying node to prolong the network time but also dynamic transmission power control mechanism for further energy saved. The node
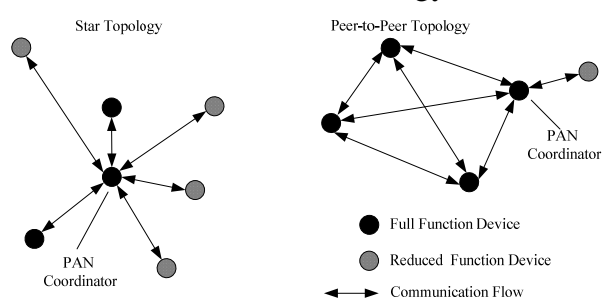

Figure 1. Two topologies defined in IEEE 802.15.4.

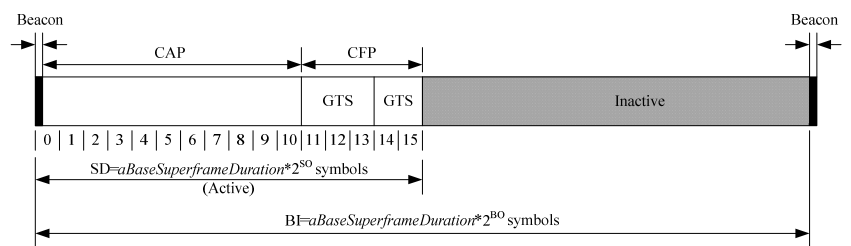

Figure 2. An example of a superframe structure in the star topology.

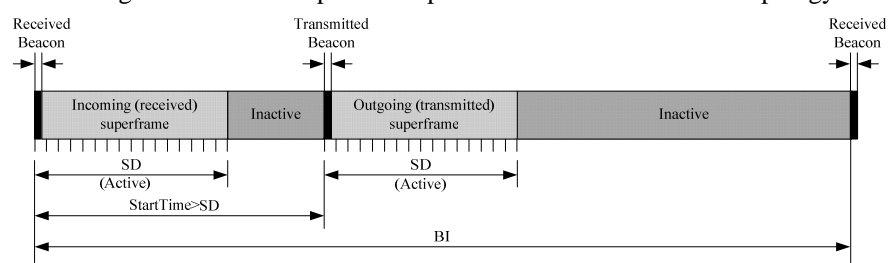

Figure 3. The relationship between incoming and outgoing superframes. begins to request relay when its residual energy is lower than a predefined energy threshold and then the coordinator will choose a proper relaying node for the node. Accordingly, the network topology is switched from one-hop to multi-hop and adjustment of the superframe is needed to support this change. In the entire communication process, dynamic transmission power control algorithm is performed based on the feedback information - RSSI from the receiver. According to the offset degree of the received RSSI value from the target RSSI threshold range, different adjustment strategies are chosen to adjust the transmission power level. Integrating relay, superframe adjustment and dynamic power control, EMAC devotes to maximizing the network lifetime.

\section{B. Dynamic Power Control Algorithm in EMAC}

In EMAC, when the RSSI value of a packet which the coordinator or the relaying node received isn't in the target RSSI threshold range [ $R_{L}, R_{H}$ ], the dynamic power control is triggered. Based on the offset degree of the received RSSI value (hereinafter denoted by $R$ ) from the $\left[R_{L}, R_{H}\right.$ ], different adjustment strategies are performed for choosing an optimal power level.

When the received $R$ is bigger than $R_{H}$, the link quality is regarded as good. Let $\alpha=R-R_{H} / R_{H}-R_{L}$, the parameter $\alpha$ denotes the offset degree of the received $R$ which is greater than $R_{H}$ from the $\left[R_{L}, R_{H}\right.$ ]. The adjustment strategy is stated as follows.

(a) When $0<\alpha=R-R_{H} / R_{H}-R_{L} \leq \alpha_{t}$ (a constant can be set according to the practical applications), it means that the offset degree is small. In this case, the coordinator notifies the sensor node by the feedback mechanism to decrease its transmission power level gradually with a fixed step $l_{c}$ until $R$ satisfies the inequality $R_{L}<R<R_{H}$.

(b) When $\alpha=R_{L}-R / R_{H}-R_{L}>\alpha_{t}$, it means that the offset degree is greater. In this case, the coordinator or the relaying node informs the node by the feedback mechanism to decrease its next transmission power level first by $l_{1}=[\alpha]\left(R_{H}-R_{L}\right)$, subsequently the node decreases its transmission power level at a time by $l_{2}=l_{3}=\cdots \cdots=l_{c}$ until $R$ satisfies the inequality $R_{L}<R<R_{H}$.

In the same way, when the received $R$ is lower than $R_{L}$, let the parameter $\beta=R_{L}-R / R_{H}-R_{L}$ denotes the offset degree of the received $R$ from $\left[R_{L}, R_{H}\right.$ ]. The adjustment strategy of the transmission power control is the same with the above method.

In conclusion, the dynamic power control algorithm in EMAC adjusts the transmission power level with a fixed step length or variable step length based on the value of offset 
degree. By this method, the sensor node can obtain an optimal transmission power level in fewer steps and energy consumption is saved.

\section{Relay Procedure}

Besides the power control mechanism, the protocol also employs relay for energy saving. Initially, we assume that all the sensor nodes working on different power levels communicate with the coordinator node through IEEE 802.15.4 star-type network and the superframe is the same with the example depicted in Fig. 2. We set $P_{i}$ to be the ratio of the residual battery power to the initial battery power for node $i$. When $P_{i}$ drops below a predefined threshold $P_{t h r}$, node $i$ begins to ask relaying nodes for help. Then the relaying node provides relay service for node $i$. Therefore, the network topology is changed from one-hop to multi-hop, and the superframe is modified to support this change. In EMAC, the main operations consist of relay request, relay response and superframe adjustment.

\section{a) Relay request}

When node $i$ in the network detects its current $P_{i}$ below $P_{t h r}$, it initiates relay request to inform the coordinator of its energy shortage (ES) by piggyback information or an exclusive frame named ES frame.

\section{b) Relay response}

During the relay response, the coordinator will select a proper relaying node for the ES node in the next modified superframe as shown in Fig. 4. The modified superframe consists of five parts: beacon, neighbor discovery period (NDP), relay decision period (RDP), CAP and CFP. The coordinator transmits the beacon frame in the first time slot and notifies what ES nodes are in the network. Through the beacon frame listening, the ES node knows at which time slot it can broadcast its relay request (RREQ) frame in NDP. The RREQ frame is used to find energy-sufficient neighbors and the FFD nodes with sufficient energy (hereinafter NES FFD for short) in the network keep awake in NDP. After the NDP, the RDP is followed. The first time slot in RDP is used for the coordinator to broadcast the notification frame (NTF). Through the NTF, each ES FFD obtains its transmission sequence in the RREP part and then replies a RREQ which contains the information of its current $P_{i}$ and a list of addresses of the devices that it has just heard during the NDP. At the end of the RDP, the WBAN coordinator broadcasts the relay decision (RDCS) frame to tell the ES nodes the corresponding addresses of their relaying nodes. The CAP and CFP of the modified superframe are worked as the IEEE 802.15.4 superframe.

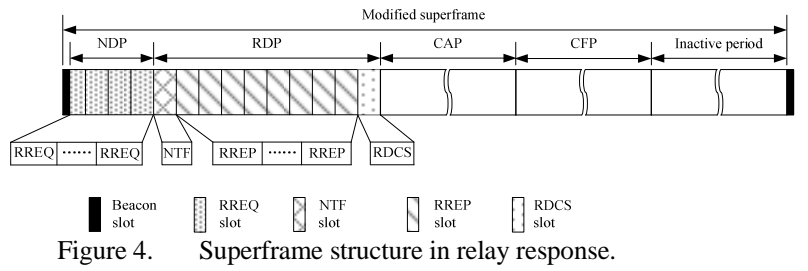

After the relaying nodes are chosen, the ES nodes will communicate with its relaying node using an optimal power level. For the NES FFD node which is selected as a relaying node, it will communicate not only with the WBAN coordinator but also with the corresponding ES node.

\section{c) Superframe adjustment}

After the relaying nodes have been determined for the ES nodes, the network topology is switched from the one-hop to the multi-hop and the superframe is changed to the example as illustrated in Fig. 3. The WBAN coordinator supports the incoming superframe and interacts with its child nodes in the active period. During the outgoing superframe, the relaying nodes act as the ordinary coordinator and interact with the ES node (the relayed nodes). Therefore, the multi-hop superframe structure is formed by the continuous overlap between the WBAN superframe and the outgoing superframes from the relaying nodes.

In short, the EMAC integrates the dynamic power control mechanism with adaptive topology adjustment to maximize the network lifetime as long as possible.

\section{PERFormance EVAluation}

\section{A. Simulation Scenario}

In this section, we have implemented IEEE 802.15.4 MAC and EMAC on the platform of MIRAI-SF [10]. Considering the heterogeneous medical applications in WBAN, we demonstrate the performance of our protocol using the scenario on human body as shown in Fig. 5. In the simulation, totally six sensor nodes are used to monitor EEG, heart rate, ECG, body temperature, pulse oximeter and respiratory rate respectively. All the sensors access the channel in CAP, and the initial energy is set to be 0.5J. In addition, regarding the power level that involved in the dynamic power control, the data from CC1000 chip [11] is adopted to offer different power levels for sensor nodes.

\section{B. Simulation Results}

Fig. 6 depicts the network lifetime as a function of energy threshold. The network lifetime here is defined as the time duration from the simulation beginning to the moment when the first sensor node runs out of its energy. It can be seen that the network lifetime of EMAC is higher than IEEE 802.15.4 obviously. For EMAC, the network lifetime increases with energy threshold and decreases later. The peak performance is achieved when the energy threshold equals to 0.6 with $106 \%$ of performance gain in network lifetime. This is because that a too low or too high energy threshold will lead to a shorter relay service. Therefore the peak performance will be achieved at a proper energy threshold.

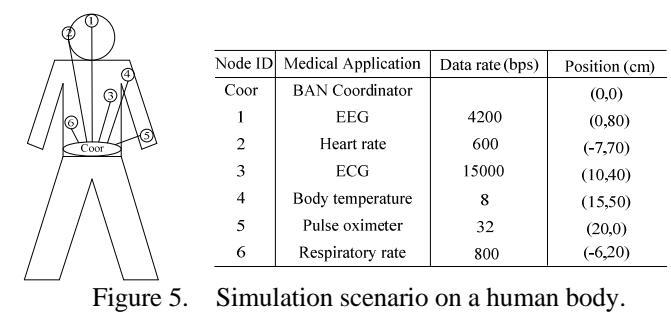




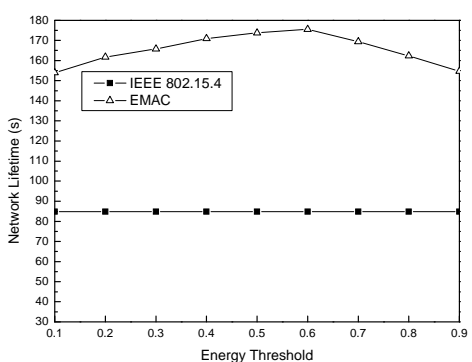

Figure 6. Network lifetime versus energy threshold.

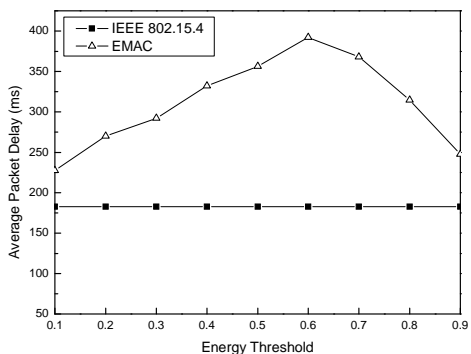

Figure 7. Average packet delay versus energy threshold.

The average end-to-end delay as a function of the energy threshold is plotted in Fig. 7. It is shown that for the EMAC the average packet delay increases with energy threshold and decreases later, and the peak value is achieved at the same energy threshold 0.6 . This is because that relay will lead to the increase of the average delay and the relay service time reaches the maximum value when the energy threshold equals to 0.6.

Furthermore, the performance of average energy consumption per packet is evaluated as shown in Fig. 8. It is observed that the average energy consumption per packet of EMAC is lower than that of IEEE 802.15.4. It can also be seen that the average energy consumption per packet of EMAC increases with the energy threshold and decreases later. The peak value is achieved when the energy threshold equals to 0.6. This is due to the fact that multiple forwarding of the packets in relay state consumes more energy and the energy threshold impacts on the relay service time.

\section{CONCLUSIONS}

In order to prolong the network lifetime, we propose an Enhanced MAC (EMAC) protocol for WBANs in this paper. The EMAC integrates relay, adaptive topology adjustment and power control to save energy. Performance evaluation is conducted in the human body scenario and the simulation results demonstrate that EMAC significantly prolongs the network lifetime. Compared with IEEE 802.15.4 MAC, the network lifetime of EMAC can be extended by $106 \%$ at most. In the future, we will consider improving EMAC in the light of specific duty cycle conditions of the devices to increase the efficiency of the protocol.

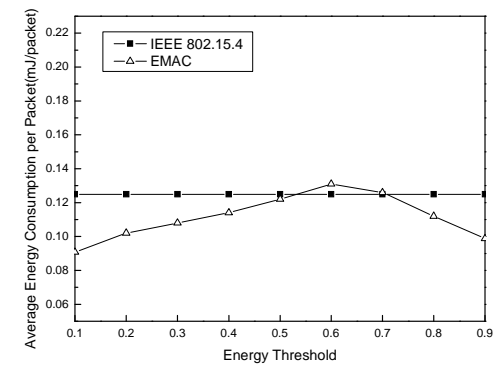

Figure 8. Average energy consumption per packet versus energy threshold.

\section{ACKNOWLEDGMENT}

This work was supported by National Natural Science Foundation of China (61271176), National Science and Technology Major Project (2013ZX03005007), Special Research Fund of State Key Laboratory (ISN1102003), Open Research Fund of National Mobile Communications Research Laboratory, Southeast University (2012D01), and the 111 Project (B08038).

\section{REFERENCES}

[1] G. Yang, Body Sensor Networks. Springer-Verlag London Ltd, 1st Edition, London, 2006.

[2] A. Boulis, D. Smith, D. Miniutti, L. Libman and Y. Tselishchev, "Challenges in body area networks for healthcare: the MAC," IEEE Commun.Mag., vol.50, no.5, pp. 100-106, May, 2012.

[3] H. Su and X. Zhang, "Battery-dynamics driven TDMA MAC protocols for wireless body-area monitoring networks in healthcare applications,” IEEE J. Sel. Areas in Commun., vol.27, no.4, pp. 424434, May, 2009.

[4] C. Li, B. Hao and K. Zhang, "A novel medium access control protocol with low delay and traffic adaptivity for wireless body area networks,” J. Med. Syst.,vol.35, no.5, pp. 1265-1275, October, 2011.

[5] H. Kwon and S. Lee, "Energy-efficient multi-hop transmission in body area networks," in Proc. of 2009 IEEE 20th International Symposium on Personal, Indoor and Mobile Radio Communications, pp. 2142-2146,Tokyo, Japan, September 13-16, September, 2009.

[6] E. Reusens, W. Joseph, B. Latre, B. Braem, G. Vermeeren, E. Tanghe, L. Martens, I. Moerman and C. Blondia, "Characterization of on-body communication channel and energy efficient topology design for wireless body area networks,” IEEE Trans. Inf. Technol. Biomed., vol.13, no.6, pp. 933-945, November, 2009.

[7] M. Quwaider, J. Rao and S. Biswas, "Body-posture-based dynamic link power control in wearable sensor networks," IEEE Commun. Mag., vol.48, no.7, pp. 134-142, July, 2010.

[8] S. Xiao, A. Dhamdhere, V. Sivaraman and A. Burdett, "Transmission power control in body area sensor networks for healthcare monitoring,” IEEE J Sel Areas in Commun, vol.27, no.1, pp.37-48, January, 2009.

[9] IEEE 802.15.4, Wireless Medium Access Control (MAC) and Physical Layer (PHY) Specifications for Low-Rate Wireless Personal Area Networks (WPANs), Sept. 2006.

[10] http://www2.nict.go.jp/w/w122/old/mt/b192/miraisf/overview_e.html.

[11] Single chip very low power RF transceiver. http://www.ti.com/lit/ds/symlink/cc1000.pdf. 